OPEN ACCESS

Edited by: Janos G. Filep,

Université de Montréal, Canada

Reviewed by:

Viswanathan Natarajan, University of Illinois at Chicago,

United States

Amiram Ariel,

University of Haifa, Israel

*Correspondence:

Remo Castro Russo

remo@ufmg.br

Andrea Don

andrea.doni@humanitasresearch.t

${ }^{\dagger}$ These authors contributed equally to this work

Specialty section:

This article was submitted to Molecular Innate Immunity, a section of the journal

Frontiers in Immunology

Received: 05 March 2021 Accepted: 02 June 2021 Published: 21 June 2021

Citation:

Doni A, Mantovani A, Bottazzi B and Russo RC (2021) PTX3 Regulation of Inflammation, Hemostatic Response,

Tissue Repair, and Resolution of

Fibrosis Favors a Role in Limiting Idiopathic Pulmonary Fibrosis.

Front. Immunol. 12:676702. doi: 10.3389/fimmu.2021.676702

\section{PTX3 Regulation of Inflammation, Hemostatic Response, Tissue Repair, and Resolution of Fibrosis Favors a Role in Limiting Idiopathic Pulmonary Fibrosis}

\author{
Andrea Doni ${ }^{1 *+}$, Alberto Mantovani ${ }^{1,2,3 \dagger}$, Barbara Bottazzi ${ }^{1+}$ and Remo Castro Russo ${ }^{4+}$ \\ 1 Unit of Advanced Optical Microscopy, Department of Immunology and Inflammation, Humanitas Clinical and Research \\ Center IRCCS, Milan, Italy, ${ }^{2}$ Department of Biomedical Sciences, Humanitas University of Milan, Milan, Italy, ${ }^{3}$ The William \\ Harvey Research Institute, Queen Mary University of London, London, United Kingdom, ${ }^{4}$ Laboratory of Pulmonary \\ Immunology and Mechanics, Department of Physiology and Biophysics, Institute of Biological Sciences, Universidade Federal \\ de Minas Gerais, Belo Horizonte, Brazil
}

PTX3 is a soluble pattern recognition molecule (PRM) belonging to the humoral innate immune system, rapidly produced at inflammatory sites by phagocytes and stromal cells in response to infection or tissue injury. PTX3 interacts with microbial moieties and selected pathogens, with molecules of the complement and hemostatic systems, and with extracellular matrix (ECM) components. In wound sites, PTX3 interacts with fibrin and plasminogen and favors a timely removal of fibrin-rich ECM for an efficient tissue repair. Idiopathic Pulmonary Fibrosis (IPF) is a chronic and progressive interstitial lung disease of unknown origin, associated with excessive ECM deposition affecting tissue architecture, with irreversible loss of lung function and impact on the patient's life quality. Maccarinelli et al. recently demonstrated a protective role of PTX3 using the bleomycin (BLM)-induced experimental model of lung fibrosis, in line with the reported role of PTX3 in tissue repair. However, the mechanisms and therapeutic potential of PTX3 in IPF remained to be investigated. Herein, we provide new insights on the possible role of PTX3 in the development of IPF and BLM-induced lung fibrosis. In mice, PTX3-deficiency was associated with worsening of the disease and with impaired fibrin removal and subsequently increased collagen deposition. In IPF patients, microarray data indicated a down-regulation of PTX3 expression, thus suggesting a potential rational underlying the development of disease. Therefore, we provide new insights for considering PTX3 as a possible target molecule underlying therapeutic intervention in IPF.

Keywords: PTX3, inflammation, hemostasis, resolution, fibrosis, IPF, humoral immunity 


\section{INTRODUCTION}

\section{Role of PTX3 in Humoral Innate Immunity}

The pentraxin family is an ancient group of evolutionarily conserved proteins belonging to humoral innate immunity that act as pattern recognition molecules (PRM). PTX3, the prototype of the long pentraxins arm, differs from the short pentraxins $C$ reactive protein (CRP) and serum amyloid $\mathrm{P}$ component (SAP/ PTX2) in molecular structure, gene organization, cellular source, and recognized ligands. PTX3 is rapidly produced by mononuclear phagocytes or stromal cells, including mesenchymal, smooth muscle, and endothelial cells (ECs) (1-5), in response to primary proinflammatory cytokines (IL-1 $\beta$ and TNF- $\alpha$ ), TLR agonists, microbial components (LPS or Outer membrane protein-A) and microbes. The molecule acts as an opsonin during infections, facilitating phagocytosis and activating the complement cascade (6). Genetic evidence in mice (7) and humans (8-15) suggests that PTX3 plays an essential role in resistance against selected pathogens, in particular A. fumigatus. In addition, PTX3 is induced in response to tissue injury and, through the interaction with the complement system and ECM components, plays non-redundant roles in tissue repair and cancer $(1,16)$. The relevance of PTX3 in the assembly of the cumulus oophorous was the first evidence of its role in ECM. Female subfertility associated with PTX3-deficiency $(2,6)$ also emphasizes the importance of this protein in ECM assembly and remodeling.

Inflammation activates various tissue response cascades that lead to ECM re-organization, removal of ECM debris, and clearance of apoptotic cells, thus favoring tissue healing. In this context, PTX3 is involved in the turnover of fibrin-rich deposits at wound sites after tissue injury, and consequent collagen deposition (16). Furthermore, when the stimuli persist or the resolution program is broken or stumbled, the inflammatory response may become chronic, impacting tissue remodeling and PTX3 expression. Macrophages are susceptible to the inflammatory environment and are key cells to modulate this system through PRMs. During the early inflammatory phase, M1-polarized macrophages accumulate and orchestrate the inflammatory response. The subsequent switch to an M2phenotype is crucial for resolving inflammation and tissue repair (17-19). M2-macrophages contribute to tissue homeostasis, dampening inflammation, scavenging ECM debris, and participating in tissue remodeling and repair $(19,20)$. On the other hand, apoptotic cells generated during chronic inflammation trigger the resolution, with significant changes in macrophage functions. Opsonization of apoptotic cells by PTX3 promotes their recognition by macrophages and subsequent efferocytosis (21), contributing to diverse M2-phenotypes switching (6) and regulating IL-10 and TGF$\beta 1$ production (22). Thus, PTX3 may play a homeostatic role in orchestrating tissue adaptation by coordinating leukocyte migration, resolution, and tissue healing.

PTX3 has been considered an essential regulator of airway mucosal surface homeostasis (23) and is useful as a disease or prognostic marker. In addition, the protein exerts a role in lung immunity against immunological dangers such as respiratory infections, allergy, tissue damage, and malfunction (24). As a humoral mediator of innate immunity, PTX3 opsonizes pulmonary pathogens promoting the clearance by phagocytosis and triggers the mucosal immune response to fungal or bacterial infections and respiratory viruses. Furthermore, PTX3 also has relevant roles in non-infectious pulmonary diseases. Altogether, PTX3 exerts multiple roles in respiratory diseases. However, its involvement in the development of IPF remains poorly explored.

\section{PTX3 AND LUNG FIBROSIS: LESSONS FROM EXPERIMENTAL MODELS OF PULMONARY FIBROSIS}

Bleomycin (BLM) is vastly used to investigate the mechanisms involved in lung fibrosis in mice, and also in the selection of therapeutic drugs for IPF, including Pirfenidone and Nintedanib $(25,26)$. Several studies have addressed the relevance of PTX3 in different models of pulmonary fibrosis (27-29). Despite the observation that BLM induces PTX3 expression in murine models of lung fibrosis $(27,29)$, a paucity of information exists on the functional role of the protein in this model. It has been shown that PTX3 promotes murine fibrocyte differentiation dependent on Fc $\gamma \mathrm{RI}$ in vitro (28). In vivo, PTX3 is localized in fibrotic areas, and its distribution is associated with collagen deposition in lung parenchyma (28) and with macrophage infiltration at sites of fibrogenesis (29), revealing an interplay with macrophages during BLM-induced tissue fibrogenesis. Taking advantage of transgenic mice overexpressing PTX3 (Tie2-PTX3), it was recently shown that the protein could limit lung fibrosis, reducing collagen deposition and fibroblast activation and decreasing leukocyte recruitment (29). Using the BLM-induced fibrosis model $(3.75 \mathrm{mg} / \mathrm{Kg}$, i.n.), we confirmed macrophage $\left(\mathrm{Ly}_{6} \mathrm{C}^{+} \mathrm{CD} 115^{+} \mathrm{CD} 11 \mathrm{~b}^{+}\right)$accumulation concomitantly with a progressive increase of PTX3 lung levels (Figure 1A), as previously described (30). Soon after BLM (2-4 days), RT-PCR showed an increased expression of M1-macrophage genes, followed later on (8-16 days) by an increased expression of M2-macrophage genes (Figure 1B). Therefore, increased PTX3 lung content is temporarily associated with a macrophage M2-polarization preceding the pulmonary fibrosis.

In the same model, $\mathrm{Ptx}^{-/-}$mice showed reduced survival (Figure 1C) and accentuated weight loss (Figure 1C). Corroborating the results by Maccarinelli et al. (29), lungs from $\mathrm{Ptx}^{-1-}$ mice showed hemorrhagic areas (day-14)(Figure 1C) and increased fibrosis, as assessed by total collagen content (day-22) (Figure 1D). In $P t x 3^{-/-}$mice lung homogenates (Figure 1E), fibrosis was not associated with differences in IL-4, IL-10, IL-6, and CCL2, thus indicating the independence of PTX3 in the regulation of inflammation $(1,2,31)$, while we found increased TGF- $\beta 1$ in $P t \times 3^{--}$ mice (Figure 1E)(day-14). As described in several models of vascular pathology or tissue repair $(16,32)$, PTX3 controls the thrombotic response by influencing platelet activation and degranulation. Therefore, it is tempting to speculate that a specific increase in lung TGF- $\beta 1$ may be due to a local release derived by platelet degranulation. PTX3 was found localized in the damaged alveolar epithelium and interstitial ECM associated with PDGFR $\alpha^{+}$ 


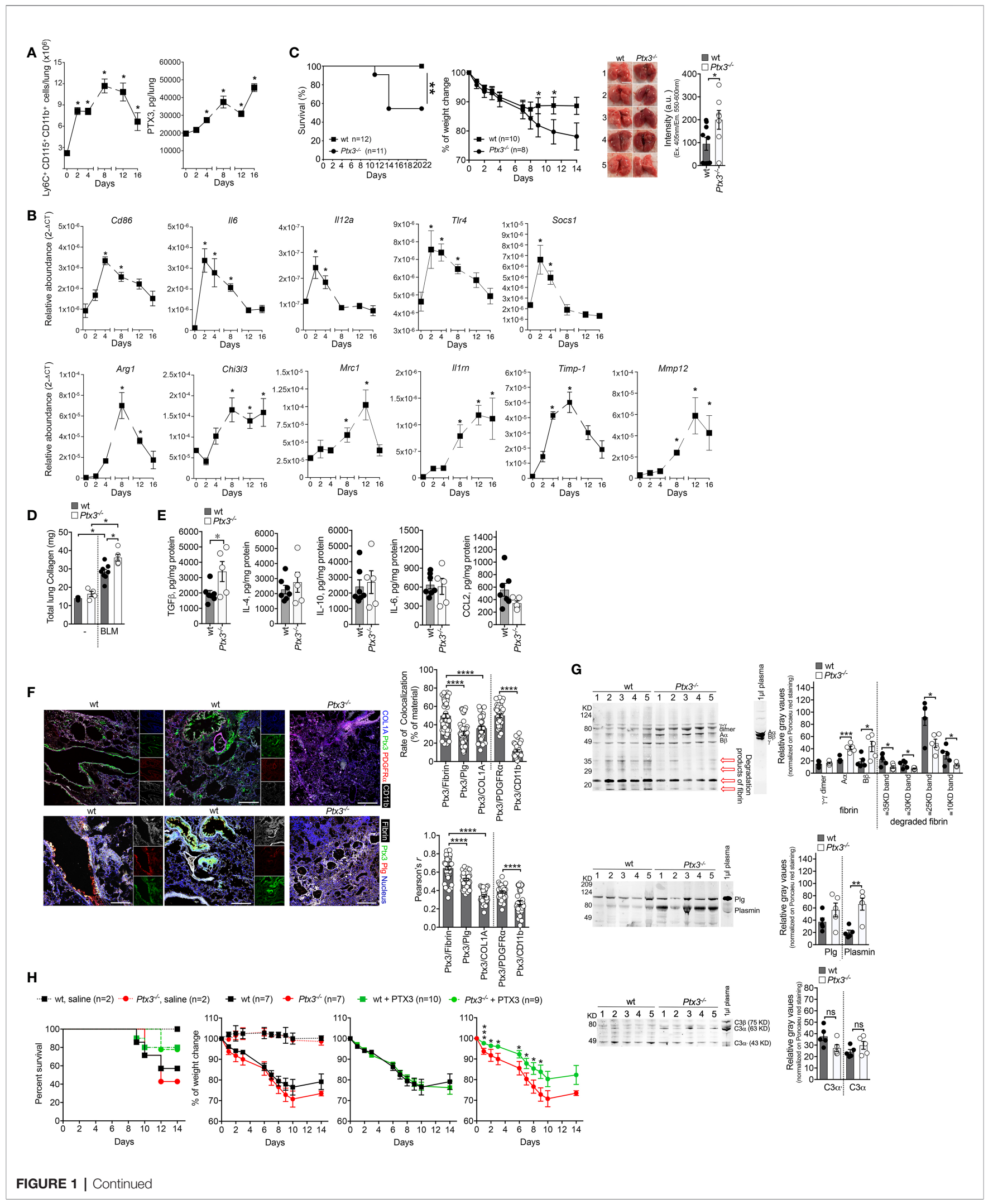




\begin{abstract}
FIGURE 1 | PTX3 protects mice from BLM-induced pulmonary fibrosis. A model of lung fibrosis was induced by BLM instillation (3.75 mg/Kg, i.n.) in Wild Type (WT) and $p t \times 3^{-1}$ mice (A-E). (A, left), kinetic of macrophage influx in the lungs of WT mice after BLM by FACS analysis. (A, right), kinetic of PTX3 lung content assessed by ELISA (PTX3 DuoSet® Kit ELISA DY2166, R\&D Systems) in tissue homogenates. (B), Transcription analysis of M1 genes (Cd86-Mm00444543_m1, II6Mm99999064_m1, Il12a-Mm99999066_m1, Trr4-Mm00445274_m1, Socs1-Mm00782550_s1) and M2 genes (Arg1-Mm00475988_m1, Chi3/3-Mm00657889_mH, Mrc1-Mm01329362_m1, II1rn-Mm00446185_m1, Timp1-Mm00441818_m1, Mmp12-Mm00500554_m1) markers of macrophage polarization by TaqMan probes (Applied Biosystems) at different days after BLM treatment. (C, D), Susceptibility of Ptx $3^{-/-}$mice to lung fibrosis induced by BLM instillation. (C, left) survival curve of mice (100\% of WT and $54.5 \%$ of Ptx3-/- mice), as defined by humane end-points (e.g., weight loss of more than $25 \%$ of initial body weight, anorexia, excessive decrease in activity, shaggy hair, diarrhea, urinary retention, breathing difficulties). ${ }^{*} P=0.01$; Log-rank test. (C, middle) monitoring of weight loss. ${ }^{*} P<0.05$; unpaired t-test). (C, right) representative photographs showing the appearance of lung parenchyma and quantification of autofluorescence intensity (excitation $405 \mathrm{~nm}$; emission collection at 550-60nm; CLARIOstar Microplate Reader, BMG Labtech) typically associated to hemoglobin in lung lysates of wt $(n=9)$ and Ptx ${ }^{-1}(\mathrm{n}=7)$ mice (day 14). * $P=0.05$, unpaired t-test. (D) lung collagen content after 21 days assessed by Sircol assay. (E), measurement of TGF- $\beta_{1}$, IL-4, IL-10, IL-6, and CCL2 in lung lysates of wt $(n=7)$ and $P t \times 3^{-1-}(n=5)$ mice (day 14 after BLM treatment) by ELISA (R\&D Systems). ${ }^{*} P=0.05$, unpaired t-test. (F), confocal microscopy analysis on lung specimens (10um) from WT mice ( $n=7) 14$ days after BLM treatment. (F, upper panels) localization of PTX3 (green), Collagen I (blue), PDGFR $\alpha^{+}($red)

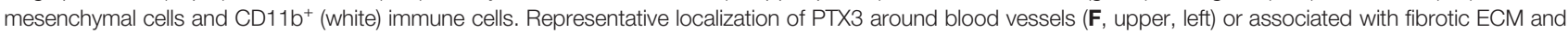
damaged epithelium (F, upper, middle). (F, lower panels) colocalization of PTX3 (green) with fibrin (white) and plasminogen (red) in fibrotic lung associated with blood vessels (F, lower, left) or ECM and damaged epithelium (F, lower, middle). Blue, nuclei. Lungs obtained from Ptx $3^{-/-}$mice were used as control (Upper and lower

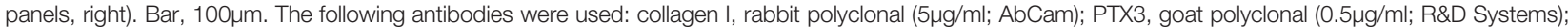

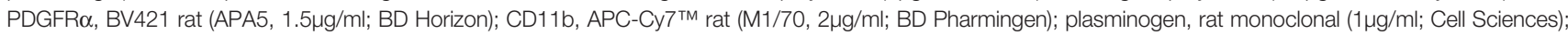

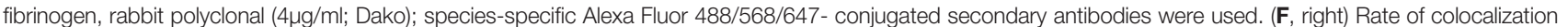
(\% of material; Fiji software) of PTX3 signal with fibrin, plasminogen, collagen I (COL1A), PDGFR $\alpha$, and CD11b and relative Pearson's correlation coefficient. Mean \pm

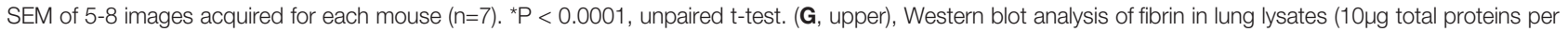
lane on $10 \%$ SDS-PAGE) of WT $(n=5)$ and $P t \times 3^{-/}(n=5)$ mice at day 7 . A polyclonal rabbit anti-fibrinogen was used $(3 \mu \mathrm{gg} / \mathrm{ml}$; Agilent/DAKO). $1 \mu \mathrm{l}$ of basal mouse plasma in ACD-A (Anticoagulant Citrate Dextrose Solution) was used as a control for fibrinogen; $1 \mu \mathrm{l}$ of mouse plasma-ACD incubated with thrombin (1U/ml; $1 \mathrm{~h}$ ) was used as a control for fibrin. A typical band pattern of fibrin ( $\mathrm{A} \alpha$; $\mathrm{B} \beta, \gamma-\gamma$ dimer) is indicated in the fibrin control and lung lysates. Red arrows, lower molecular weight bands corresponding to degraded fibrin. (G, upper, right), quantification of fibrin bands as relative gray values (Fiji software) on Ponceau red staining. ${ }^{* \star *} P<0.005$, ${ }^{\star} P<0.5$; unpaired t-test. (G, middle), Western blot analysis of plasminogen and relative band quantification as gray values (Fiji software) in same lysates (50 $\mu$ g total proteins per lane on 10\% SDS-PAGE, G, middle, right). A polyclonal goat anti-plasminogen was used (0.5 $\mathrm{mg} / \mathrm{ml}$; R\&D Systems). The molecular weight of plasminogen and plasmin activation bands are indicated. (G, lower), Western blot analysis of the complement component C3 in the same lysates (10 $\mu \mathrm{g}$ total proteins per lane on 10\% SDS-PAGE) and relative band quantification as gray values (Fiii software, G, lower, right). A polyclonal goat anti-human/mouse C3 and activation fragments (1:3000; Merck-Millipore) was used. (H) Effect of PTX3 administration in BLM-induced lung fibrosis. One experiment was performed. Human recombinant PTX3 (50 $\mathrm{\mu g} / \mathrm{mouse})$ was injected i.p. one day after BLM (5 mg/Kg, i.n.) treatment in WT mice. Survival (H, left) (Ptx3-/- mice from $42.8 \%$ to $77.8 \%$, and WT mice from 57.1 to $80 \%$ of survival) and body weight (H, 3 panels right) were recorded until day 14 . Curves referring to weight loss are shown compared to untreated WT and $P t \times 3^{-/-}$mice ( $\mathbf{H}$, first left) or separated by genotype and compared with the correspondent treated group $\left(\mathbf{H}\right.$, right). ${ }^{*} P<0.05$; unpaired t-test. All results were expressed as mean \pm SEM. Normalized data were analyzed by One-Way ANOVA with Tukey post-test, using the software GraphPad Prism 8.0. Differences were considered significant at $\mathrm{P}<0.05$.
\end{abstract}

mesenchymal cells, and $P t x 3^{--}$mice showed increased interstitial fibrin deposition and subsequent fibrotic scarring in ALI (acute lung injury) model (5). Similarly, in the BLM-induced fibrotic lungs (day-14), PTX3 is localized in damaged epithelium and areas of ECM rich in collagen-I, preferentially associated with PDGFR $\alpha^{+}$ mesenchymal cells rather than recruited $\mathrm{CD}_{11 \mathrm{~b}^{+}}$cells (day-7) (Figure 1F). Moreover, PTX3 is localized around the blood vessels (Figure 1F). Thus, in line with evidence obtained in different experimental models of lung injury and repair $(16,33$, 34), PTX3 plays a non-redundant and protective role during BLMinduced pulmonary fibrosis in mice.

PTX3 promotes arterial thrombosis (32) at wound sites during injury-induced thrombotic response and promotes healing by interacting with plasminogen, favoring timely fibrin removal in acidic microenvironments $(5,16,33,35)$. As presented in Figure 1F, in the BLM-induced fibrotic lungs PTX3 colocalized with fibrin deposits in ECM and damaged epithelium closely associated with plasminogen. A similar colocalization was observed in the endothelium of blood vessels possibly associated with coagulation sites (day-7) (Figure 1F). Coagulation proteases are recognized to exert profibrotic cellular effects via activation of protease-activated receptors (PARs) (36). Fibrinolysis is also an essential prerequisite for subsequent tissue remodeling processes leading to efficient repair (37-40). Therefore, analysis of fibrin and plasminogen content in lung lysates would address whether a defective turnover in fibrin removal was present in $P t \times 3^{-/-}$mice. As assessed by Western blot, lungs of $P t x 3^{-/-}$mice showed increased fibrin deposition and decreased fragments of fibrin degradation at the inflammatory phase (day-7)(Figure 1G). Differences in plasminogen deposition and plasmin formation (day-7)(Figure 1G) observed in the same lung homogenates suggest an impairment in ECM turnover of fibrin, possibly at the bases of a subsequent increased lung fibrosis. No evidence of PTX3 regulation on complement activation was observed in this model, as no differences were found in C3 deposition in the lung (Figure 1G). Several reports showed that coagulation cascade elements are involved in lung fibrosis (41). The deficiency of components of the fibrinolytic system caused exacerbated lung injury associated with defective clearance of necrotic tissue and augmented fibrin deposition and fibrosis (42). Lung fibrosis was reverted by overexpression of plasminogen activator genes (38, 39). Therefore, the disruption of fibrin removal and altered ECM turnover with collagen deposition appeared as the central mechanism underlying the phenotypes associated with $P t \times 3^{-1-}$ mice in response to BLM, corroborating the observations from other models of organ damage $(5,33,35)$. TGF- $\beta 1$-mediated elevated PAI-1 levels and defective fibrinolysis have significant fibrotic consequences for tissue repair $(43,44)$. Interestingly, TGF- $\beta 1$ was found to down-regulate PTX3 at mRNA and 
protein levels in granuloma (45) and mesenchymal cells (our results, data not shown). TGF- $\beta 1$ increased ECM deposition promoting transcription of collagen and protease inhibitors, including tissue inhibitors of metalloproteases (TIMPs) and PAI-1 (46). Concomitantly, TGF- $\beta 1$ decreases the secretion of proteases responsible for ECM degradation, including activators of plasminogen $(43,44)$, thus increasing the overall production of ECM proteins (47). Therefore, PTX3 down-regulation by TGF- $\beta 1$ could inhibit the removal of the fibrin matrix and increases ECM deposition during experimental fibrosis.

The evidence obtained from the BLM-induced lung fibrosis model prompted us to evaluate the possible therapeutic effects of PTX3 treatment in the disease. In a first preliminary experiment, shown in Figure 1H, a single i.p. injection of recombinant PTX3 $(50 \mu \mathrm{g} / \mathrm{mouse})$ one day after BLM $(5 \mathrm{mg} / \mathrm{Kg}$, i.n. $)$ was sufficient to increase survival of $P t \times 3^{-/-}$mice, with a weak effect even in WT mice survival (Figure 1H). PTX3 treatment also attenuated the weight loss in $\mathrm{Ptx}^{-/-}$mice but not in WT mice challenged with BLM (Figure 1G), suggesting a potential therapeutic effect of PTX3 in pulmonary fibrosis. However, the actual evaluation of potential PTX3 treatment requires more detailed pharmacological studies on the doses and administration routes.

\section{MECHANISMS OF PTX3 IN IDIOPATHIC PULMONARY FIBROSIS: INTERPRETING DATA FROM IPF}

IPF is a chronic and lethal Interstitial Lung Disease characterized by fibroproliferation of unknown origin and insensitive to therapy, associated with excessive ECM deposition in the pulmonary parenchyma (48-50). Clinical signs include progressive loss of lung volume, increased respiratory effort, and abnormal gas exchange, leading to respiratory collapse (48-50). Currently, some hypotheses have been proposed about its controversial origin. Among them, clinical data have revealed a close correlation between pulmonary fibrosis and the profile of inflammatory mediators released by immune cells (19, $51,52)$. Data supports that pulmonary fibrosis is the final result of previous alveolitis with excessive scarring $(19,52,53)$. Current knowledge about IPF has been derived from detailed pathological analysis of human lung samples that elucidated its unique morphological characteristics, together with observations derived from animal models of disease $(26,52,54,55)$. New insights raised from genetic and transcriptomic studies on IPF samples have given a better comprehension of the molecular and cellular mechanisms determining the lung phenotype of IPF and patient therapeutics $(25,54,56)$.

The physiological role of PTX3 in IPF remains to be elucidated. However, present results and previous evidence suggest a role of PTX3 in lung repair in experimental models of lung injury (16), an association of PTX3 and Primary Graft Dysfunction (PGD) in IPF recipients after lung transplant (57), or a potential role of PTX3 produced by fibroblasts and bronchial epithelial cells in fibrocyte differentiation in vitro (28). In the lung tissue samples obtained from IPF patients, PTX3 was found associated with fibrotic areas of ECM, epithelium, and alveolar leukocytes (28). In order to gain a deeper insight into this association, we analyzed microarray data of lung samples from IPF (GEO database: GSE32537) using Phantasus (58), a web application for visual and interactive gene expression analysis (https://genome.ifmo.ru/phantasus). Besides upregulated genes related to fibrosis (COL1A1, FGFR2, TIMP2, and TGF- $\beta 2 / 3$; Figures 2A, C), increased expression of M2-macrophage and inhibition of M1-macrophage related genes were found in IPF samples (Figures 2A, C), similarly to WT mice exposed to BLM (Figure 1B). Moreover, IL-10 and PTX3 expression were downregulated in IPF in this set of lung microarray (Figure 2A), as shown by normalized data (Figure 2C). PLAUR and SERPINE1 , genes belonging to the fibrinolytic system and related to tissue repair, were also down-regulated (Figures $\mathbf{2 B}, \mathbf{C}$ ). Thus, PTX3 gene down-regulation in lung samples may be related to active IPF pathology. This recapitulates the mouse phenotype observed in vivo, with aberrant collagen deposition in $P t \times 3^{-/-}$mice [Figure 1 (29)], supporting the possibility that endogenous PTX3 exerts a protective role and may be involved in IPF disease. Therefore, PTX3 down-regulation could be part of a TGF- $\beta 1$ regulatory program leading to fibrinolysis inhibition and ECM increased deposition, both determinants of IPF pathogenesis and progression.

\section{THERAPEUTIC OPPORTUNITIES OF PTX3 IN THE CONTEXT OF IPF: WHAT MECHANISMS WOULD BE INVOLVED?}

Data reported by Maccarinelli et al. (29) and our results suggest that IPF may be related to a low expression of PTX3 in lung tissue. Therefore, we could consider restoring PTX3 levels exogenously (as summarized in Figure 1G) as a possible therapeutic approach for IPF. Different roles of PTX3 could be considered:

\section{PTX3 as a Negative Modulator of Chronic Airway Inflammation in IPF}

Chronic inflammation can lead to an imbalance in soluble factors production and leukocyte recruitment, turning the healing response into a pathological fibrotic response (52). Chronic neutrophilic airway inflammation occurs in IPF and airway neutrophilia, mainly due to CXCL8 produced by alveolar macrophages (59), and predicts mortality of IPF patient (60). PTX3 regulates neutrophils influx through interaction with P-selectin expressed on the surface of ECs $(31,61)$, thus, exogenous PTX3 may dampen neutrophil influx into IPF airways (Figure 2D). Moreover, soluble PTX3 derived from Human Umbilical Cord Blood-Derived Mesenchymal Stem Cells (UCB-MSCs) has anti-inflammatory effects in ALI, as shown in a model of neonatal hyperoxia-induced lung injury in rats. Similarly, adoptive transfer of MSC from WT but not from $\mathrm{Ptx} 3^{-/-}$mice improved oxygenation with reduced lung collapse and neutrophils (33), shaping the differentiation of anti-inflammatory macrophages (62). On the other hand, Dendritic cells (DCs), activated by the phagocytosis of apoptotic cells, are described to sustain chronic lung inflammation in IPF (63). Nevertheless, in the presence of PTX3, DCs failed to internalize apoptotic cells (21), thus suggesting that 
A
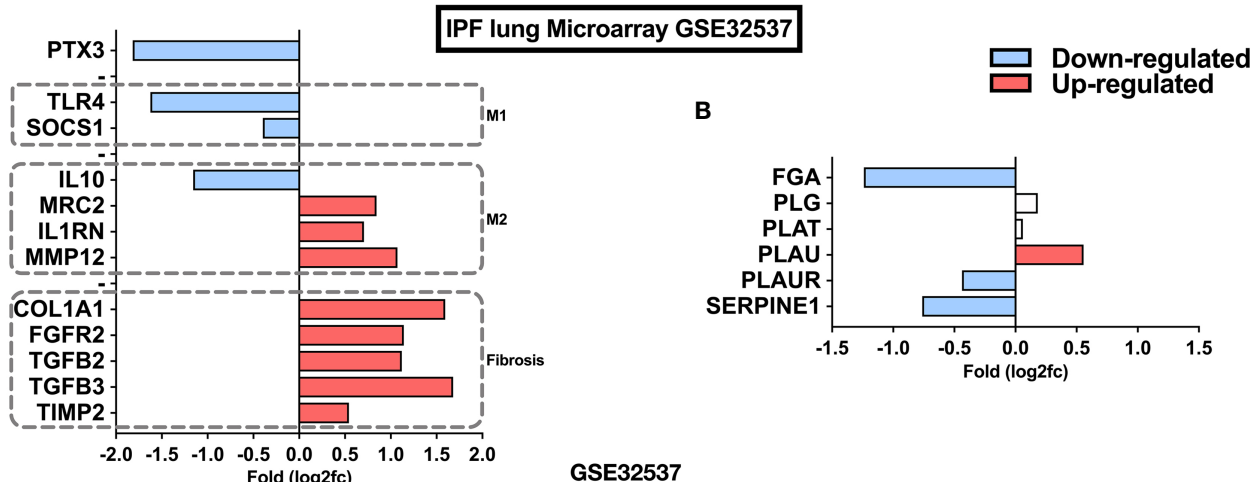

B Up-regulated

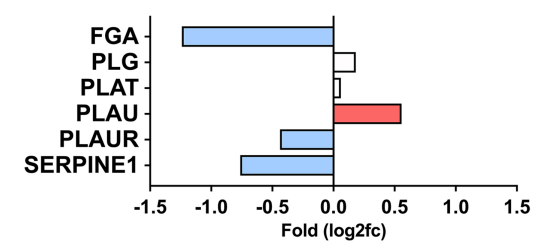

GSE32537

\begin{tabular}{|l|c|c|c|c|c|c|c|c|}
\hline Gene Symbol & Gene ID & id & Mean & logFC & AveExpr & t & P.value & adj.P.Value \\
\hline PTX3 & 5806 & 8083594 & 0.63574 & -1.8181 & 0.74146 & -7.0405 & $3.2250 \mathrm{e}-11$ & $1.7547 \mathrm{e}-10$ \\
\hline TLR4 & 7099 & 8157524 & 0.071815 & -1.6243 & 0.088094 & -10.750 & $1.8988 \mathrm{e}-21$ & $5.2936 \mathrm{e}-20$ \\
\hline SOCS1 & 8651 & 7999423 & -0.027459 & -0.39781 & -0.040678 & -2.0485 & 0.041856 & 0.059050 \\
\hline IL10 & 3586 & 7923907 & 0.13283 & -1.1573 & 0.16465 & -6.8779 & $8.1196 \mathrm{e}-11$ & $4.1557 \mathrm{e}-10$ \\
\hline MRC2 & 9902 & 8009040 & -0.071049 & 0.84345 & -0.15301 & 5.5922 & $7.5337 \mathrm{e}-8$ & $2.5350 \mathrm{e}-7$ \\
\hline IL1RN & 3557 & 8044574 & 0.021796 & 0.70672 & -0.028085 & 4.5895 & 0.0000079653 & 0.000020288 \\
\hline MMP12 & 4321 & 7951297 & 0.31147 & 1.0723 & 0.30598 & 6.3163 & $1.7876 \mathrm{e}-9$ & $7.5498 \mathrm{e}-9$ \\
\hline COL1A1 & 1277 & 8016646 & -0.23474 & 1.5947 & -0.23526 & 12.839 & $1.0231 \mathrm{e}-27$ & $9.1658 \mathrm{e}-26$ \\
\hline FGFR2 & 2263 & 7936734 & -0.10101 & 1.1425 & -0.14084 & 6.8839 & $7.8509 \mathrm{e}-11$ & $4.0249 \mathrm{e}-10$ \\
\hline TGFB2 & 7042 & 7909789 & -0.10138 & 1.1194 & -0.14667 & 7.9968 & $1.1234 \mathrm{e}-13$ & $9.0536 \mathrm{e}-13$ \\
\hline TGFB3 & 7043 & 7980316 & -0.30323 & 1.6805 & -0.32534 & 11.353 & $3.0673 \mathrm{e}-23$ & $1.1943 \mathrm{e}-21$ \\
\hline TIMP2 & 7077 & 8018966 & 0.0038976 & 0.54221 & -0.050177 & 3.3805 & 0.00087474 & 0.0016350 \\
\hline FGA & 2243 & 8103311 & 0.34674 & -1.2403 & 0.38802 & -7.8177 & $3.3326 \mathrm{e}-13$ & $2.4982 \mathrm{e}-12$ \\
\hline PLG & 5340 & 8123259 & -0.0027377 & 0.18178 & 0.027985 & 0.93537 & 0.35076 & 0.40349 \\
\hline PLAT & 5327 & 8150509 & -0.042061 & 0.059786 & -0.029896 & 0.34840 & 0.72792 & 0.76445 \\
\hline PLAU & 5328 & 7928429 & 0.030326 & 0.55692 & -0.0052238 & 3.3176 & 0.0010838 & 0.0019962 \\
\hline PLAUR & 5329 & 8037374 & -0.096047 & -0.43784 & -0.16066 & -2.8687 & 0.0045771 & 0.0076021 \\
\hline SERPINE1 & 5054 & 8135069 & 0.12340 & -0.76302 & 0.16283 & -5.0865 & $8.5665 \mathrm{e}-7$ & 0.0000024820 \\
\hline & & & & & & & \\
\hline
\end{tabular}

D

\section{Therapeutic opportunities of PTX3 in the context of IPF:}

Chronic Pulmonary Inflammation and Fibrosis Resolution of Chronic Pulmonary Inflammation and Fibrosis by PTX3

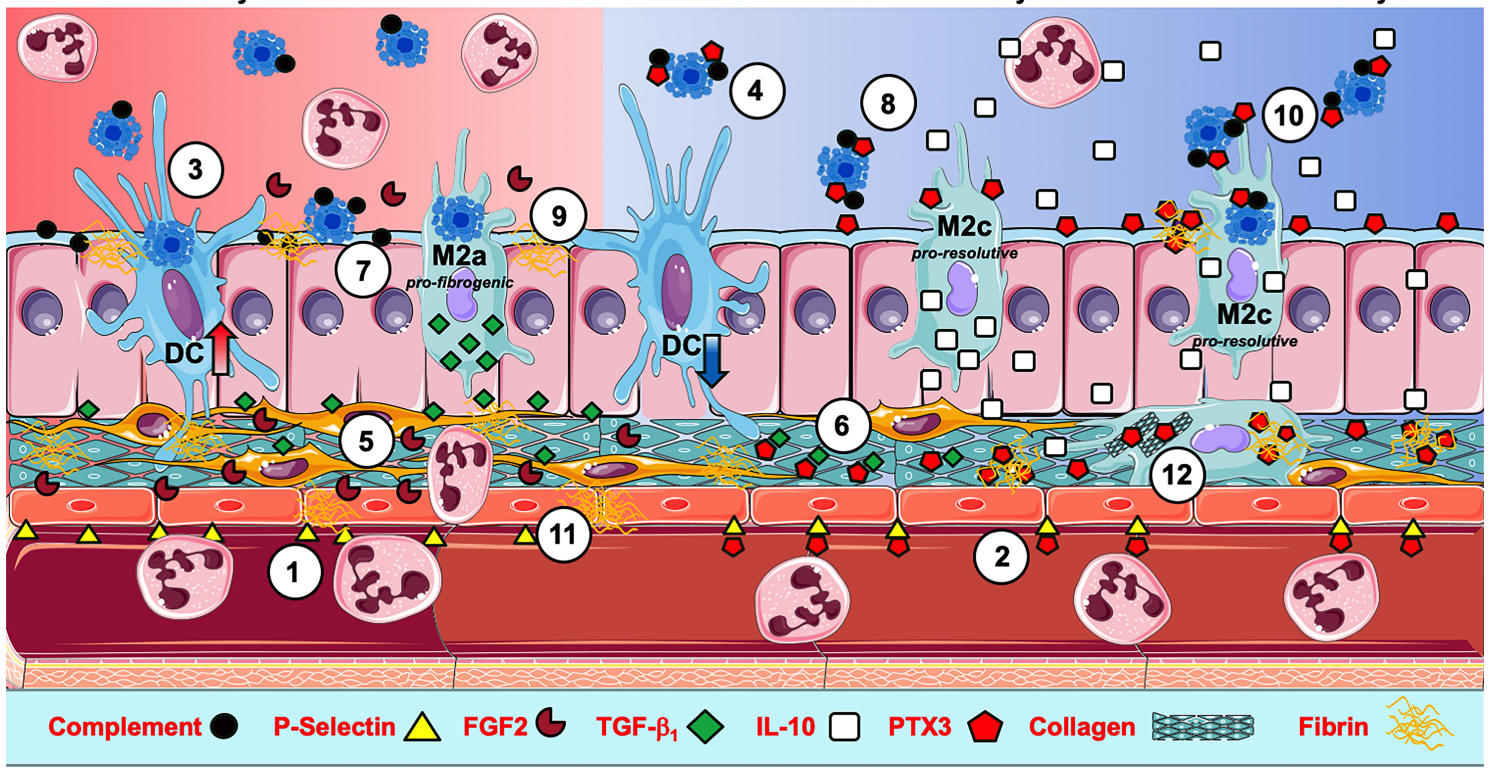

FIGURE 2 | Continued 


\begin{abstract}
FIGURE 2 | Impact of PTX3 in Idiopathic Pulmonary Fibrosis and therapeutic opportunities. Microarray analysis of lung samples from IPF ( $\mathrm{n=119)}$ and healthy ( $\mathrm{n=50}$ individuals from GEO database: GSE32537 were analyzed by Phantasus (58) (https://genome.ifmo.ru/phantasus). (A) Lung expression of PTX3, fibrogenic markers as COL1A1, FGFR2, TGFB2, TGFB3 and TIMP-2, M1 (SOCS1 and TLR4) and M2 (IL-10, MRC2, IL1RN, and MMP-12) macrophage polarization markers (B) Expression of coagulation cascade FGA (Fibrinogen alpha-chain precursor), PLG (Plasminogen precursor), PLAT (Tissue-type Plasminogen Activator precursor), PLAU (Urokinase-type Plasminogen Activator precursor), PLAUR (Urokinase Plasminogen Activator Receptor) and SERPINE1 (PAI-1 Plasminogen Activator Inhibitor1) in IPF and health samples. (C) Data table with analysis from GEO database GSE32537 analyzed by Phantasus according to the instructions for the use of the application. Differences were considered significant at $P$ value $<0,05$. (D) Possible mechanisms and therapeutic opportunities of PTX3 in the context of IPF. IPF is characterized by a reduced PTX3 production (red background), however PTX3 may act as an anti-inflammatory as well as a pro-resolutive modulator of chronic pulmonary inflammation and fibrosis in IPF (blue background) at different levels: (1) Neutrophil influx is facilitated through the interaction with P-selectin expressed on the surface of ECs, (2) PTX3 could antagonize endothelial P-selectin, dampening neutrophil influx during chronic pulmonary inflammation; (3) the abundance of apoptotic cells in airways from IPF is related to DC phagocytosis and activation that sustain chronic lung inflammation, (4) PTX3 may block apoptotic cell internalization and consequent inflammation; (5) FGF2 activates fibroblasts and ECs, (6) however PTX3 interacts with FGF2 reducing its availability for binding to FGFR2 on fibroblasts and consequent fibrosis; (7) complement and apoptotic cell deposition in the lungs lead to chronic inflammation, (8) on the other side PTX3 may act as a scavenger preventing the excessive deposition of both complement components and apoptotic cells in lungs and consequent attenuation of tissue damage and inflammation; (9) Alveolar macrophages from IPF display defective efferocytosis and increased TGF- $\beta 1$ production, contributing to tissue fibrogenesis, (10) while PTX3 may enhance macrophage efferocytosis and M2 polarization and resolution of inflammation by IL-10; (11) Finally, defective PTX3 production in IPF may increase fibrin deposition and fibrosis, (12) but PTX3 could contribute to the resolution of fibrosis, interacting with fibrin-clots and disorganized collagen fibers in the lung parenchyma, supporting fibrinolysis and clearance of ECM debris by macrophage phagocytosis, promoting lung tissue healing and repair.
\end{abstract}

PTX3 may prevent chronic pulmonary inflammation in IPF (Figure 2D). However, whether endogenous or exogenous PTX3 acts as a tissue-protective or resilience factor stimulating lung tissue reepithelization remains unexplored.

\section{PTX3 Involvement in Resolution of Inflammation in IPF}

Efficient resolution of inflammation is crucial for the restoration of tissue integrity. PTX3 has been reported to induce the polarization of macrophages into anti-inflammatory M2 phenotype and to stimulate them to secrete the resolutive cytokine IL-10 $(62,64)$. Moreover, as mentioned above, PTX3 production by MSC reinforced M2 macrophage markers, inducing Dectin-1 and IL10 , and protecting mice from neonatal hyperoxia-induced lung injury (62). Therefore, PTX3 may contribute to the resolution of chronic inflammation in IPF via M2-macrophages enhancing IL10-dependent anti-inflammatory and resolutive functions, such as neutrophil apoptosis (Figure 2D). Nevertheless, impaired efferocytosis can result in inflammation-associated pathologies (65). Indeed, efferocytosis by alveolar macrophages is impaired in IPF samples compared with other interstitial pneumonia (66), and a dysregulated or defective efferocytosis may contribute to the pathogenesis of IPF $(65,66)$. Notably, post-efferocytotic, satiated macrophages [also termed Mres (67)] produce high levels of TGF- $\beta 1$ (68). However, this production seems to be functionally antagonized by the production of IFN- $\beta$ in these macrophages (69), that directs their anti-fibrotic phenotype $(70,71)$. Along these lines, apoptotic cell instillation after BLM attenuates lung injury (72) and induces PPAR $\gamma$, promoting lung fibrosis resolution via regulation of efferocytosis and IL-10 production (73). In this way, PTX3 recognizes apoptotic cells and may facilitate the clearance of dead or dying cells (1). Therefore, the capacity of PTX3 to affect apoptotic cell recognition and efferocytosis could represent an additional mechanism of negative regulation of chronic inflammation in IPF (Figure 2D) (66). PTX3 enhanced complement-mediated clearance of apoptotic debris. The protein is recruited by $\mathrm{C} 4$ binding protein (C4BP) on apoptotic cells reducing the deposition of the lytic C5b-9 terminal complex at sites of tissue injury (74), limiting the complement-mediated tissue damage and inflammation (1), and possibly tissue fibrosis (Figure 2D).

\section{PTX3 as a Resolutive Modulator of Tissue Fibrosis in IPF}

PTX3 interaction with plasminogen ensures the timely removal of fibrin deposits in the inflammatory ECM of the lung, allowing for the proper sequence of processes leading to efficient tissue repair. TGF- $\beta 1$, the major fibrogenic molecule involved in the mechanisms of excessive ECM deposition in pulmonary fibrosis, negatively regulates PTX3 and ECM-degrading molecules (e.g., MMPs, uPA) and up-regulates TIMPs and PAI- $1(45,75)$. Thus, defective PTX3mediated fibrinolysis may represent a key mechanism underlying the development of the disease. Therefore, TGF- $\beta 1$ induces suppression of PTX3-mediated fibrinolysis and may represent another mechanism underlying the development of IPF. In other contexts of tissue repair $(16,33,35)$, PTX3 administration reversed the defective fibrinolysis associated with PTX3-deficiency and this may represent an important activity underlying PTX3 role in regulating the evolution towards fibrotic scarring of the lung (Figure 2D). PTX3 interacts with FGF2 and modulates the FGFR2-dependent vascularization of tumors and FGFR2mediated smooth muscle cell proliferation and artery restenosis $(76,77)$. The expression of FGF2/FGFR2 axis is elevated in IPF samples (78), and FGFR2-dependent signaling is involved in pulmonary fibrosis $(76,79)$. Therefore, a down-regulation of PTX3 in IPF may represent a possible failure to antagonize these fibrotic pathways (Figure 2D). Finally, PTX3 also interacts with collagens and our own preliminary results indicate a possible involvement of collagen remodeling by mesenchymal cells, thus suggesting an effect in removing the excess of ECM from tissue parenchyma (Figure 2D) and hence in promoting tissue healing.

\section{CONCLUDING AND REMARKS}

Failure to control the overlapping events leading to the healing process is the cause of the functional tissue replacement by fibrous scar. Growing evidences indicate that abnormalities in pathways involving fibroblast activation and coagulation cascade drive abnormal fibroproliferation and progressive replacement of lung parenchyma by collagen $(37,80)$. Understanding the molecules 
involved in these pathways during aberrant wound repair may predict new targets and therapeutic intervention strategies. PTX3, besides being an essential fluid-phase PRM of the innate immune system, is involved in the healing at wound sites, favoring timely fibrinolysis through the interaction with fibrin and plasminogen. Results reported by Maccarinelli and colleagues and our observations indicate a protective and regulatory role of PTX3 in BLM-induced lung fibrosis models of lung injury. Besides IPF, other pathological conditions can result in pulmonary fibrosis, last but not least the persistent post-COVID syndrome $(81,82)$. Many questions remain open, starting from the therapeutic effect of PTX3 to the mechanisms involved in the protective role of the protein and the relationships of PTX3 with the homeostasis of the airway epithelium and with the collagen fibers of the ECM. Little is known about the effect of IPF therapy with Pirfenidone/Nintedanib on PTX3. Further clinical studies will be necessary to answer these and many other questions. Thus, in summary, we assessed the involvement of PTX3 in pulmonary fibrosis. Based on the literature and recent data, we propose that PTX3 may have a physiological and protective role during IPF, interacting with various circuits and representing a potential therapeutic target, acting as a pro-resolutive molecule in the context of pulmonary fibrosis.

\section{DATA AVAILABILITY STATEMENT}

The datasets presented in this study can be found in online repositories. The names of the repository/repositories and accession number(s) can be found below: https://www.ncbi. nlm.nih.gov/geo/, GSE32537.

\section{REFERENCES}

1. Garlanda C, Bottazzi B, Magrini E, Inforzato A, Mantovani A. PTX3, a Humoral Pattern Recognition Molecule, in Innate Immunity, Tissue Repair, and Cancer. Physiol Rev (2018) 98:623-39. doi: 10.1152/physrev.00016.2017

2. Magrini E, Mantovani A, Garlanda C. The Dual Complexity of PTX3 in Health and Disease: A Balancing Act? Trends Mol Med (2016) 22:497-510. doi: 10.1016/j.molmed.2016.04.007

3. Doni A, Stravalaci M, Inforzato A, Magrini E, Mantovani A, Garlanda C, et al. The Long Pentraxin PTX3 as a Link Between Innate Immunity, Tissue Remodeling, and Cancer. Front Immunol (2019) 10:712. doi: 10.3389/fimmu.2019.00712

4. Manfredi AA, Rovere-Querini P, Bottazzi B, Garlanda C, Mantovani A. Pentraxins, Humoral Innate Immunity and Tissue Injury. Curr Opin Immunol (2008) 20:538-44. doi: 10.1016/j.coi.2008.05.004

5. Doni A, Garlanda C, Mantovani A. Innate Immunity, Hemostasis and Matrix Remodeling: PTX3 as a Link. Semin Immunol (2016) 28:570-7. doi: 10.1016/ j.smim.2016.10.012

6. Erreni M, Manfredi AA, Garlanda C, Mantovani A, Rovere-Querini P. The Long Pentraxin PTX3: A Prototypical Sensor of Tissue Injury and a Regulator of Homeostasis. Immunol Rev (2017) 280:112-25. doi: 10.1111/imr.12570

7. Garlanda C, Hirsch E, Bozza S, Salustri A, De Acetis M, Nota R, et al. NonRedundant Role of the Long Pentraxin PTX3 in Anti-Fungal Innate Immune Response. Nature (2002) 420:182-6. doi: 10.1038/nature01195

8. Olesen R, Wejse C, Velez DR, Bisseye C, Sodemann M, Aaby P, et al. DCSIGN (CD209), Pentraxin 3 and Vitamin D Receptor Gene Variants Associate With Pulmonary Tuberculosis Risk in West Africans. Genes Immun (2007) 8:456-67. doi: 10.1038/sj.gene.6364410

9. Cunha C, Aversa F, Lacerda JF, Busca A, Kurzai O, Grube M, et al. Genetic PTX3 Deficiency and Aspergillosis in Stem-Cell Transplantation. N Engl J Med (2014) 370:421-32. doi: 10.1056/NEJMoa1211161

\section{ETHICS STATEMENT}

The animal study was reviewed and approved by by the Italian Ministry of Health (protocol approval n. 803/2015-PR).

\section{AUTHOR CONTRIBUTIONS}

$\mathrm{AD}, \mathrm{AM}, \mathrm{BB}$, and RCR designed the discussion, conclusions and wrote the manuscript. All authors contributed to the article and approved the submitted version.

\section{FUNDING}

RCR is grateful to the Conselho Nacional de Desenvolvimento Científico e Tecnológico (CNPq, Brazil) under Grant Agreement No. 312839/2020-0. AD, BB, and AM are grateful to the Italian Association for Cancer Research (AIRC; grant number IG23465) and the Italian Space Agency (ASI MARS-PRE Project, grant number DC-VUM 2017-006) for financial support.

\section{ACKNOWLEDGMENTS}

We would like to thank the professors Dr. Flavio Almeida Amaral and Dr. Jerome Baron from UFMG, for the helpful give-a-take. Schematic Figure (Figure 2D) used images from Servier Medical Art, licensed under a Creative Common Attribution 3.0 Generic License. (http://smart.servier.com/).

10. Cunha C, Monteiro AA, Oliveira-Coelho A, Kuhne J, Rodrigues F, Sasaki SD, et al. Ptx3-Based Genetic Testing for Risk of Aspergillosis After Lung Transplant. Clin Infect Dis (2015) 61:1893-4. doi: 10.1093/cid/civ679

11. Wojtowicz A, Lecompte TD, Bibert S, Manuel O, Rueger S, Berger C, et al. PTX3 Polymorphisms and Invasive Mold Infections After Solid Organ Transplant. Clin Infect Dis (2015) 61:619-22. doi: 10.1093/cid/civ386

12. Fisher CE, Hohl TM, Fan W, Storer BE, Levine DM, Zhao LP, et al. Validation of Single Nucleotide Polymorphisms in Invasive Aspergillosis Following Hematopoietic Cell Transplantation. Blood (2017) 129:2693-701. doi: 10.1182/blood-2016-10-743294

13. Herrero-Sanchez MC, Angomas EB, de Ramon C, Telleria JJ, Corchete LA, Alonso S, et al. Polymorphisms in Receptors Involved in Opsonic and Nonopsonic Phagocytosis, and Correlation With Risk of Infection in Oncohematology Patients. Infect Immun (2018) 86(12):e00709-18. doi: 10.1128/IAI.00709-18

14. Campos CF, Leite L, Pereira P, Vaz CP, Branca R, Campilho F, et al. PTX3 Polymorphisms Influence Cytomegalovirus Reactivation After Stem-Cell Transplantation. Front Immunol (2019) 10:88. doi: 10.3389/fimmu.2019.00088

15. Chiarini M, Sabelli C, Melotti P, Garlanda C, Savoldi G, Mazza C, et al. PTX3 Genetic Variations Affect the Risk of Pseudomonas Aeruginosa Airway Colonization in Cystic Fibrosis Patients. Genes Immun (2010) 11:665-70. doi: 10.1038/gene.2010.41

16. Doni A, Musso T, Morone D, Bastone A, Zambelli V, Sironi M, et al. An Acidic Microenvironment Sets the Humoral Pattern Recognition Molecule PTX3 in a Tissue Repair Mode. J Exp Med (2015) 212:905-25. doi: 10.1084/jem.20141268

17. Watanabe S, Alexander M, Misharin AV, Budinger GRS. The Role of Macrophages in the Resolution of Inflammation. J Clin Invest (2019) 129:2619-28. doi: 10.1172/JCI124615

18. Mantovani A, Biswas SK, Galdiero MR, Sica A, Locati M. Macrophage Plasticity and Polarization in Tissue Repair and Remodelling. J Pathol (2013) 229:176-85. doi: 10.1002/path.4133 
19. Vannella KM, Wynn TA. Mechanisms of Organ Injury and Repair by Macrophages. Annu Rev Physiol (2017) 79:593-617. doi: 10.1146/annurevphysiol-022516-034356

20. Mantovani A, Sica A, Sozzani S, Allavena P, Vecchi A, Locati M. The Chemokine System in Diverse Forms of Macrophage Activation and Polarization. Trends Immunol (2004) 25:677-86. doi: 10.1016/j.it.2004.09.015

21. Rovere P, Peri G, Fazzini F, Bottazzi B, Doni A, Bondanza A, et al. The Long Pentraxin PTX3 Binds to Apoptotic Cells and Regulates Their Clearance by Antigen-Presenting Dendritic Cells. Blood (2000) 96:4300-6. doi: 10.1182/ blood.V96.13.4300

22. Baruah P, Propato A, Dumitriu IE, Rovere-Querini P, Russo V, Fontana R, et al. The Pattern Recognition Receptor PTX3 is Recruited at the Synapse Between Dying and Dendritic Cells, and Edits the Cross-Presentation of Self, Viral, and Tumor Antigens. Blood (2006) 107:151-8. doi: 10.1182/blood-2005-03-1112

23. Smole U, Kratzer B, Pickl WF. Soluble Pattern Recognition Molecules: Guardians and Regulators of Homeostasis at Airway Mucosal Surfaces. Eur J Immunol (2020) 50:624-42. doi: 10.1002/eji.201847811

24. Balhara J, Koussih L, Zhang J, Gounni AS. Pentraxin 3: An Immuno-Regulator in the Lungs. Front Immunol (2013) 4:127. doi: 10.3389/fimmu.2013.00127

25. Tashiro J, Rubio GA, Limper AH, Williams K, Elliot SJ, Ninou I, et al. Exploring Animal Models That Resemble Idiopathic Pulmonary Fibrosis. Front Med (Lausanne) (2017) 4:118. doi: 10.3389/fmed.2017.00118

26. Miles T, Hoyne GF, Knight DA, Fear MW, Mutsaers SE, Prele CM. The Contribution of Animal Models to Understanding the Role of the Immune System in Human Idiopathic Pulmonary Fibrosis. Clin Transl Immunol (2020) 9:e1153. doi: 10.1002/cti2.1153

27. Della Latta V, Cabiati M, Burchielli S, Frenzilli G, Bernardeschi M, Cecchettini A, et al. Lung Inflammation After Bleomycin Treatment in Mice: Selection of an Accurate Normalization Strategy for Gene Expression Analysis in an ExVivo and in-Vitro Model. Int J Biochem Cell Biol (2017) 88:145-54. doi: 10.1016/j.biocel.2017.05.016

28. Pilling D, Cox N, Vakil V, Verbeek JS, Gomer RH. The Long Pentraxin PTX3 Promotes Fibrocyte Differentiation. PloS One (2015) 10:e0119709. doi: 10.1371/journal.pone.0119709

29. Maccarinelli F, Bugatti M, Schuind AC, Ganzerla S, Vermi W, Presta M, et al. Endogenous Long Pentraxin 3 Exerts a Protective Role in a Murine Model of Pulmonary Fibrosis. Front Immunol (2021) 12:617671. doi: 10.3389/ fimmu.2021.617671

30. Russo RC, Savino B, Mirolo M, Buracchi C, Germano G, Anselmo A, et al. The Atypical Chemokine Receptor ACKR2 Drives Pulmonary Fibrosis by Tuning Influx of CCR2(+) and CCR5(+) Ifngamma-Producing Gammadeltat Cells in Mice. Am J Physiol Lung Cell Mol Physiol (2018) 314:L1010-25. doi: 10.1152/ ajplung.00233.2017

31. Deban L, Russo RC, Sironi M, Moalli F, Scanziani M, Zambelli V, et al. Regulation of Leukocyte Recruitment by the Long Pentraxin PTX3. Nat Immunol (2010) 11:328-34. doi: 10.1038/ni.1854

32. Bonacina F, Barbieri SS, Cutuli L, Amadio P, Doni A, Sironi M, et al. Vascular Pentraxin 3 Controls Arterial Thrombosis by Targeting Collagen and Fibrinogen Induced Platelets Aggregation. Biochim Biophys Acta (2016) 1862:1182-90. doi: 10.1016/j.bbadis.2016.03.007

33. Mauri T, Zambelli V, Cappuzzello C, Bellani G, Dander E, Sironi M, et al. Intraperitoneal Adoptive Transfer of Mesenchymal Stem Cells Enhances Recovery From Acid Aspiration Acute Lung Injury in Mice. Intensive Care Med Exp (2017) 5:13. doi: 10.1186/s40635-017-0126-5

34. Han B, Haitsma JJ, Zhang Y, Bai X, Rubacha M, Keshavjee S, et al. Long Pentraxin PTX3 Deficiency Worsens LPS-Induced Acute Lung Injury. Intensive Care Med (2011) 37:334-42. doi: 10.1007/s00134-010-2067-2

35. Cappuzzello C, Doni A, Dander E, Pasqualini F, Nebuloni M, Bottazzi B, et al. Mesenchymal Stromal Cell-Derived PTX3 Promotes Wound Healing via Fibrin Remodeling. J Invest Dermatol (2016) 136:293-300. doi: 10.1038/JID.2015.346

36. Wygrecka M, Kwapiszewska G, Jablonska E, von Gerlach S, Henneke I, Zakrzewicz D, et al. Role of Protease-Activated Receptor-2 in Idiopathic Pulmonary Fibrosis. Am J Respir Crit Care Med (2011) 183:1703-14. doi: 10.1164/rccm.201009-1479OC

37. Idell S. Coagulation, Fibrinolysis, and Fibrin Deposition in Acute Lung Injury. Crit Care Med (2003) 31:S213-20. doi: 10.1097/01.CCM.0000057846.21303.AB

38. Eitzman DT, McCoy RD, Zheng X, Fay WP, Shen T, Ginsburg D, et al. Bleomycin-Induced Pulmonary Fibrosis in Transgenic Mice That Either Lack or Overexpress the Murine Plasminogen Activator Inhibitor-1 Gene. J Clin Invest (1996) 97:232-7. doi: 10.1172/JCI118396

39. Sisson TH, Hattori N, Xu Y, Simon RH. Treatment of Bleomycin-Induced Pulmonary Fibrosis by Transfer of Urokinase-Type Plasminogen Activator Genes. Hum Gene Ther (1999) 10:2315-23. doi: 10.1089/104303 49950016960

40. Bdeir K, Murciano JC, Tomaszewski J, Koniaris L, Martinez J, Cines DB, et al. Urokinase Mediates Fibrinolysis in the Pulmonary Microvasculature. Blood (2000) 96:1820-6. doi: 10.1182/blood.V96.5.1820.h8001820_1820_1826

41. Gunther A, Lubke N, Ermert M, Schermuly RT, Weissmann N, Breithecker A, et al. Prevention of Bleomycin-Induced Lung Fibrosis by Aerosolization of Heparin or Urokinase in Rabbits. Am J Respir Crit Care Med (2003) 168:135865. doi: $10.1164 / \mathrm{rccm} .2201082$

42. Swaisgood CM, French EL, Noga C, Simon RH, Ploplis VA. The Development of Bleomycin-Induced Pulmonary Fibrosis in Mice Deficient for Components of the Fibrinolytic System. Am J Pathol (2000) 157:177-87. doi: 10.1016/ S0002-9440(10)64529-4

43. de Giorgio-Miller A, Bottoms S, Laurent G, Carmeliet P, Herrick S. FibrinInduced Skin Fibrosis in Mice Deficient in Tissue Plasminogen Activator. Am J Pathol (2005) 167:721-32. doi: 10.1016/S0002-9440(10)62046-9

44. Ghosh AK, Vaughan DE. PAI-1 in Tissue Fibrosis. J Cell Physiol (2012) 227:493-507. doi: $10.1002 /$ jcp. 22783

45. Li H, Chang HM, Shi Z, Leung PCK. SNAIL Mediates TGF-Betal-Induced Downregulation of Pentraxin 3 Expression in Human Granulosa Cells. Endocrinology (2018) 159:1644-57. doi: 10.1210/en.2017-03127

46. Massague J, Wotton D. Transcriptional Control by the TGF-beta/Smad Signaling System. EMBO J (2000) 19:1745-54. doi: 10.1093/emboj/19.8.1745

47. Hall MC, Young DA, Waters JG, Rowan AD, Chantry A, Edwards DR, et al. The Comparative Role of Activator Protein 1 and Smad Factors in the Regulation of Timp-1 and MMP-1 Gene Expression by Transforming Growth Factor-Beta 1. J Biol Chem (2003) 278:10304-13. doi: 10.1074/ jbc.M212334200

48. Somogyi V, Chaudhuri N, Torrisi SE, Kahn N, Muller V, Kreuter M. The Therapy of Idiopathic Pulmonary Fibrosis: What is Next? Eur Respir Rev (2019) 28(153). doi: 10.1183/16000617.0021-2019

49. Kropski JA, Blackwell TS. Progress in Understanding and Treating Idiopathic Pulmonary Fibrosis. Annu Rev Med (2019) 70:211-24. doi: 10.1146/annurevmed-041317-102715

50. Antoniou KM, Wuyts W, Wijsenbeek M, Wells AU. Medical Therapy in Idiopathic Pulmonary Fibrosis. Semin Respir Crit Care Med (2016) 37:368-77. doi: $10.1055 / \mathrm{s}-0036-1582010$

51. Heukels P, Moor CC, von der Thusen JH, Wijsenbeek MS, Kool M. Inflammation and Immunity in IPF Pathogenesis and Treatment. Respir Med (2019) 147:79-91. doi: 10.1016/j.rmed.2018.12.015

52. Wilson MS, Wynn TA. Pulmonary Fibrosis: Pathogenesis, Etiology and Regulation. Mucosal Immunol (2009) 2:103-21. doi: 10.1038/mi.2008.85

53. Chambers RC, Mercer PF. Mechanisms of Alveolar Epithelial Injury, Repair, and Fibrosis. Ann Am Thorac Soc (2015) 12 Suppl 1:S16-20. doi: 10.1513/ AnnalsATS.201410-448MG

54. Yu G, Ibarra GH, Kaminski N. Fibrosis: Lessons From OMICS Analyses of the Human Lung. Matrix Biol (2018) 68-69:422-34. doi: 10.1016/j.matbio.2018.03.014

55. Carrington R, Jordan S, Pitchford SC, Page CP. Use of Animal Models in IPF Research. Pulm Pharmacol Ther (2018) 51:73-8. doi: 10.1016/ j.pupt.2018.07.002

56. Neumark N, Cosme CJr., Rose KA, Kaminski N. The Idiopathic Pulmonary Fibrosis Cell Atlas. Am J Physiol Lung Cell Mol Physiol (2020) 319:L887-93. doi: 10.1152/ajplung.00451.2020

57. Diamond JM, Lederer DJ, Kawut SM, Lee J, Ahya VN, Bellamy S, et al. Elevated Plasma Long Pentraxin-3 Levels and Primary Graft Dysfunction After Lung Transplantation for Idiopathic Pulmonary Fibrosis. Am J Transplant (2011) 11:2517-22. doi: 10.1111/j.1600-6143.2011.03702.x

58. Zenkova D, Kamenev V, Sablina R, Artyomov M, , Phantasus SA. Visual and Interactive Gene Expression Analysis. doi: 10.18129/B9.bioc.phantasus

59. Carre PC, Mortenson RL, King TEJr., Noble PW, Sable CL, Riches DW. Increased Expression of the Interleukin-8 Gene by Alveolar Macrophages in Idiopathic Pulmonary Fibrosis. A Potential Mechanism for the Recruitment and Activation of Neutrophils in Lung Fibrosis. J Clin Invest (1991) 88:180210. doi: 10.1172/JCI115501 
60. Kinder BW, Brown KK, Schwarz MI, Ix JH, Kervitsky A, King TEJr. Baseline BAL Neutrophilia Predicts Early Mortality in Idiopathic Pulmonary Fibrosis. Chest (2008) 133:226-32. doi: 10.1378/chest.07-1948

61. Lech M, Rommele C, Grobmayr R, Eka Susanti H, Kulkarni OP, Wang S, et al. Endogenous and Exogenous Pentraxin-3 Limits Postischemic Acute and Chronic Kidney Injury. Kidney Int (2013) 83:647-61. doi: 10.1038/ki.2012.463

62. Kim M, Kwon JH, Bae YK, Kim GH, Um S, Ha J, et al. Soluble PTX3 of Human Umbilical Cord Blood-Derived Mesenchymal Stem Cells Attenuates Hyperoxic Lung Injury by Activating Macrophage Polarization in Neonatal Rat Model. Stem Cells Int (2020) 2020:1802976. doi: 10.1155/2020/1802976

63. Marchal-Somme J, Uzunhan Y, Marchand-Adam S, Kambouchner M, Valeyre D, Crestani B, et al. Dendritic Cells Accumulate in Human Fibrotic Interstitial Lung Disease. Am J Respir Crit Care Med (2007) 176:1007-14. doi: 10.1164/rccm.200609-1347OC

64. Zhang S, Zhu YT, Chen SY, He H, Tseng SC. Constitutive Expression of Pentraxin 3 (PTX3) Protein by Human Amniotic Membrane Cells Leads to Formation of the Heavy Chain (HC)-Hyaluronan (HA)-PTX3 Complex. J Biol Chem (2014) 289:13531-42. doi: 10.1074/jbc.M113.525287

65. Kourtzelis I, Hajishengallis G, Chavakis T. Phagocytosis of Apoptotic Cells in Resolution of Inflammation. Front Immunol (2020) 11:553. doi: 10.3389/ fimmu.2020.00553

66. Morimoto K, Janssen WJ, Terada M. Defective Efferocytosis by Alveolar Macrophages in IPF Patients. Respir Med (2012) 106:1800-3. doi: 10.1016/ j.rmed.2012.08.020

67. Ariel A, Serhan CN. New Lives Given by Cell Death: Macrophage Differentiation Following Their Encounter With Apoptotic Leukocytes During the Resolution of Inflammation. Front Immunol (2012) 3:4. doi: 10.3389/fimmu.2012.00004

68. Schif-Zuck S, Gross N, Assi S, Rostoker R, Serhan CN, Ariel A. SaturatedEfferocytosis Generates Pro-Resolving CD11b Low Macrophages: Modulation by Resolvins and Glucocorticoids. Eur J Immunol (2011) 41:366-79. doi: 10.1002/eji.201040801

69. Kumaran Satyanarayanan S, El Kebir D, Soboh S, Butenko S, Sekheri M, Saadi J, et al. IFN-Beta is a Macrophage-Derived Effector Cytokine Facilitating the Resolution of Bacterial Inflammation. Nat Commun (2019) 10:3471. doi: 10.1038/s41467-019-10903-9

70. Azuma A, Li YJ, Abe S, Usuki J, Matsuda K, Henmi S, et al. Interferon-\{Beta\} Inhibits Bleomycin-Induced Lung Fibrosis by Decreasing Transforming Growth Factor-\{Beta\} and Thrombospondin. Am J Respir Cell Mol Biol (2005) 32:93-8. doi: 10.1165/rcmb.2003-0374OC

71. Butenko S, Satyanarayanan SK, Assi S, Schif-Zuck S, Sher N, Ariel A. Transcriptomic Analysis of Monocyte-Derived Non-Phagocytic Macrophages Favors a Role in Limiting Tissue Repair and Fibrosis. Front Immunol (2020) 11:405. doi: 10.3389/fimmu.2020.01003

72. Lee YJ, Moon C, Lee SH, Park HJ, Seoh JY, Cho MS, et al. Apoptotic Cell Instillation After Bleomycin Attenuates Lung Injury Through Hepatocyte Growth Factor Induction. Eur Respir J (2012) 40:424-35. doi: 10.1183/ 09031936.00096711
73. Yoon YS, Kim SY, Kim MJ, Lim JH, Cho MS, Kang JL. Ppargamma Activation Following Apoptotic Cell Instillation Promotes Resolution of Lung Inflammation and Fibrosis via Regulation of Efferocytosis and Proresolving Cytokines. Mucosal Immunol (2015) 8:1031-46. doi: 10.1038/mi.2014.130

74. Braunschweig A, Jozsi M. Human Pentraxin 3 Binds to the Complement Regulator c4b-binding Protein. PloS One (2011) 6:e23991. doi: 10.1371/ journal.pone.0023991

75. Vaughan DE. PAI-1 and TGF-beta: Unmasking the Real Driver of TGF-BetaInduced Vascular Pathology. Arterioscler Thromb Vasc Biol (2006) 26:679-80. doi: 10.1161/01.ATV.0000209949.86606.c2

76. Guzy RD, Li L, Smith C, Dorry SJ, Koo HY, Chen L, et al. Pulmonary Fibrosis Requires Cell-Autonomous Mesenchymal Fibroblast Growth Factor (FGF) Signaling. J Biol Chem (2017) 292:10364-78. doi: 10.1074/jbc.M117.791764

77. Rusnati M, Camozzi M, Moroni E, Bottazzi B, Peri G, Indraccolo S, et al. Selective Recognition of Fibroblast Growth Factor-2 by the Long Pentraxin PTX3 Inhibits Angiogenesis. Blood (2004) 104:92-9. doi: 10.1182/blood-200310-3433

78. Li L, Zhang S, Wei L, Wang Z, Ma W, Liu F, et al. FGF2 and FGFR2 in Patients With Idiopathic Pulmonary Fibrosis and Lung Cancer. Oncol Lett (2018) 16:2490-4. doi: 10.3892/ol.2018.8903

79. Kim MH, Jung SY, Song KH, Park JI, Ahn J, Kim EH, et al. A New FGFR Inhibitor Disrupts the TGF-beta1-induced Fibrotic Process. J Cell Mol Med (2020) 24:830-40. doi: 10.1111/jcmm.14793

80. Marshall RP, Bellingan G, Webb S, Puddicombe A, Goldsack N, McAnulty RJ, et al. Fibroproliferation Occurs Early in the Acute Respiratory Distress Syndrome and Impacts on Outcome. Am J Respir Crit Care Med (2000) 162:1783-8. doi: 10.1164/ajrccm.162.5.2001061

81. George PM, Wells AU, Jenkins RG. Pulmonary Fibrosis and COVID-19: The Potential Role for Antifibrotic Therapy. Lancet Respir Med (2020) 8:807-15. doi: $10.1016 / \mathrm{S} 2213-2600(20) 30225-3$

82. Oronsky B, Larson C, Hammond TC, Oronsky A, Kesari S, Lybeck M, et al. A Review of Persistent Post-Covid Syndrome (Ppcs). Clin Rev Allergy Immunol (2021) 1-9. doi: 10.1007/s12016-021-08848-3

Conflict of Interest: AM and BB are inventors of patents on pentraxin-3 and obtain royalties on related reagents.

The remaining authors declare that the research was conducted in the absence of any commercial or financial relationships that could be construed as a potential conflict of interest.

Copyright $\odot 2021$ Doni, Mantovani, Bottazzi and Russo. This is an open-access article distributed under the terms of the Creative Commons Attribution License (CC BY). The use, distribution or reproduction in other forums is permitted, provided the original author(s) and the copyright owner(s) are credited and that the original publication in this journal is cited, in accordance with accepted academic practice. No use, distribution or reproduction is permitted which does not comply with these terms. 